\title{
CSF-1 receptor signalling is governed by pre-requisite EHD1 mediated receptor display on the macrophage cell surface
}

\author{
Luke R. Cypher ${ }^{1}$, Timothy Alan Bielecki ${ }^{1}$, Lu Huang ${ }^{4}$, Wei An ${ }^{2}$, Fany Iseka ${ }^{2}$, Eric Tom ${ }^{3}$, \\ Matthew D. Storck ${ }^{1}$, Adam D. Hoppe ${ }^{4}$, Vimla Band ${ }^{1,2}$, Hamid Band ${ }^{1,2^{*}}$ \\ ${ }^{1}$ Eppley Cancer Institute for Research in Cancer \& Allied Diseases, University of Nebraska \\ Medical Center, Omaha, Nebraska, United States of America \\ ${ }^{2}$ Department of Genetics, Cell Biology, \& Anatomy, University of Nebraska Medical Center, \\ Omaha, Nebraska, United States of America \\ ${ }^{3}$ Department of Biochemistry \& Molecular Biology, University of Nebraska Medical Center, \\ Omaha, Nebraska, United States of America \\ ${ }^{4}$ Department of Chemistry and Biochemistry, South Dakota State University, Brookings, South \\ Dakota, United States of America \\ *Correspondence: \\ Hamid Band M.D., Ph.D. \\ University of Nebraska Medical Center \\ DRC II - 5064 \\ Omaha, NE 68198-5950
}

Tel: (402) 559-8572; Fax: (402) 559-4651; E-mail: hband@umnc.edu

Address for page proofs and requests for reprints:

E-mail: hband@unmc.edu (Hamid Band) 


\begin{abstract}
Colony stimulating factor-1 receptor (CSF-1R), a receptor tyrosine kinase (RTK), is the master regulator of macrophage biology. CSF-1 can bind CSF-1R resulting in receptor activation and signalling essential for macrophage functions such as proliferation, differentiation, survival, polarization, phagocytosis, cytokine secretion, and motility. CSF-1R activation can only occur after the receptor is presented on the macrophage cell surface. This process is reliant upon the underlying macrophage receptor trafficking machinery. However, the mechanistic details governing this process are incompletely understood. C-terminal Eps15 Homology Domaincontaining (EHD) proteins have recently emerged as key regulators of receptor trafficking but have not yet been studied in the context of macrophage CSF-1R signalling. In this manuscript, we utilize primary bone-marrow derived macrophages (BMDMs) to reveal a novel function of EHD1 as a regulator of CSF-1R abundance on the cell surface. We report that EHD1-knockout (EHD1-KO) macrophages cell surface and total CSF-1R levels are significantly decreased. The decline in CSF-1R levels corresponds with reduced downstream macrophage functions such as cell proliferation, migration, and spreading. In EHD1-KO macrophages, transport of newlysynthesized CSF-1R to the macrophage cell surface was reduced and was associated with the shunting of the receptor to the lysosome, which resulted in receptor degradation. These findings reveal a novel and functionally important role for EHD1 in governing CSF-1R signalling via regulation of anterograde transport of CSF-1R to the macrophage cell surface.
\end{abstract}

\title{
Keywords
}

CSF-1R, macrophage, proliferation, cell signalling, cell surface, receptor tyrosine kinase

\section{Footnote}

Abbreviations: CSF1R, Colony Stimulating Factor 1 Receptor; CSF1, Colony Stimulating Factor-1; BMDM, Bone Marrow Derived Macrophage; EHD1, Eps-15 Homology Domaincontaining protein 1; EHD1-KO, EHD1-KnockOut (4-hydroxytamoxifen induced deletion); RTK, Receptor Tyrosine Kinase; TAM, 4-Hydroxytamoxifen; FACS, Fluorescence Activated Cell Sorting; c.p.m., counts per minute; GM130, Golgi Matrix Protein (130kD); LAMP1, Lysosomal associated membrane protein 1; PFA, Paraformaldehyde; BSA, Bovine Serum Albumin 


\section{Introduction}

Cells of the monocyte-macrophage lineage play fundamental roles in integrating innate and adaptive arms of immune defense and aberrations of development or function of this cell lineage are incompatible with an effective immune system[1,2]. Macrophages are key contributors to highlyprevalent immunological diseases [3-5] such as rheumatoid arthritis, inflammatory bowel disease, and demyelinating neurological diseases[6-8]. Macrophages also play key homeostatic roles in non-immune tissues and contribute to diseases such as atherosclerosis and cancer[9-11].

Colony-stimulating factor-1 receptor (CSF-1R) is a receptor tyrosine kinase (RTK) essential for macrophage development and physiological functions, such as cell proliferation, differentiation, spreading, migration, phagocytosis and cytokine secretion [12]. Similar to other RTKs, ligand binding of Colony-stimulating factor-1 (CSF-1) activates CSF-1R by inducing conformational changes, dimerization, and trans-phosphorylation, leading to association of key cytoplasmic signalling intermediates. This activates signal transduction cascades, including the PI3-kinase/AKT, Ras/Erk, and Rho/Rac pathways[13,14].

Ligand binding (RTK activation) induces the endocytic internalization of RTKs, and this has been demonstrated for CSF-1R [15-17]. Endocytosed RTKs are targeted to the lysosome, which we and others have established to be dependent on ubiquitination via CBL-family and recognition by ESCRT complexes [15,18-21]. Internalizationand degradation of RTKs have classically been thought to be a mechanism for cellular attenuation of RTK signalling.However, activated RTKs are known to signal in endosomes and may require endocytosis to transduce specific signals [22]. Recently,Erk1/2 activation due to CSF-1R signalling has been shownto require endocytosis, while signalling at the cell surface was sufficient for STAT pathway activation [23].

Compared to intracellular trafficking of ligand-stimulated RTKs, the trafficking of unstimulated/basalRTKs is less understood. In the absence of CSF-1, CSF-1R has been shown to undergo constitutive internalization and basal recycling at a relatively low rate [24]. An understanding of the mechanisms of RTK traffic in the absence of ligand-induced activation is of fundamental importance as these mechanisms, together with the anterograde transport of newly synthesized protein, help determine the level of the activation-ready, cell surface pool of RTKs. Such mechanisms can also determine the kinetics of restoration of cell surface RTK display following ligand-induced degradation for subsequent ligand binding and activation. Yet, little is known about these basic mechanisms of RTK traffic, and CSF-1R trafficking in macrophages has not been reported.

The four members of the Eps15-homology domain-containing (EHD) protein family have emerged as regulators of cellular receptor trafficking[25]. Structurally, EHD proteins are characterized by highlyrelated primary amino acid sequencesand conserved domain structure amongst family members: a nucleotide-binding G-domain that folds similarly to the GTPase dynamin, but instead hydrolyzes ATP; coiled-coiled regions that form a membrane lipid-binding 
domain; and an $\mathrm{EH}$ domain near the $\mathrm{C}$-terminus that mediates interactions with partner proteins by binding to asparagine-proline-phenylalanine (NPF)-containing motifs [26]. In vitro studies have established that EHD proteins function similarly to dynamin as scission proteins to promote vesicular budding involved in cellular trafficking of receptors [27]. While biochemical roles of EHD proteins have been elucidated in some detail, their physiological roles are just now being unraveled.

Our laboratoryhas previously utilized mouse models to understand the physiological functions of EHD family proteins[28-34]. We have found that EHD1 deletion on a mixed C57BL/6 and 129Sv background is partially penetrant embryonic lethal and associated with defective spermatogenesis and lens developmental defects, while it is fully embryonic lethal on a predominantly C57BL/6 background [35-37]. Here, we use primary bone marrow-derived macrophages (BMDMs)with inducible EHD1-Knockout (EHD1-KO) capacity, to study the biological and physiological function of EHD1 in macrophages. Here, we report EHD1 is required for delivery and display of CSF-1Rupon the cell surface, ensuringCSF-1R activation/signalling, and downstream macrophage functional response.

\section{Materials \& Methods}

\subsection{Reagents \& Antibodies}

Bovine Serum Albumin (cat. \# A7906-100G), Paraformaldehyde (cat. \# 158127-500G), Triton X-100 (cat. \# 93418), and 4-hydroxytamoxifen (cat. \# T176-10MG) were from SigmaAldrich (St. Louis, MO). Propidium Iodide staining solution (cat. \# 00-6990-42) was from eBiosciences. Hema staining solutions (cat. \# 23-123919) were from Fisher. 3H-thymidine (cat. \# 2407001, 2.0Ci/mmol) was from MP Biomedical and [35S] (cat. \# NEG772007MC, 1175 Ci/mmol) was from Perkin Elmer. Bafilomycin A1 (cat. \# 196000) was from MilliPore. EDTAfree protease inhibitor cocktail (cat. \# 4693159001) was from Roche, ECL development reagent (cat. \# 32106) was from Thermo-Scientific. Recombinant CSF-1 (catalog \# 315-02) was from Peprotech (Rocky Hill, NJ). CFSE (cat. \# C34554), RPMI-1640 (cat. \# SH30027.02). Penicillin/streptomycin (cat. \# 15140-122) and Fetal Bovine Serum (cat. \# 10427-028; lot \# 1662765A120-01) were from Life Technologies. Antibodies: Brilliant Violet 711 conjugated anti-CSF1R and anti-CSF1R, AFS98 (cat. \# 135515) were from Biolegend; anti-CSF1R C-20 (cat. \# sc-692), anti-CSF1R G-17 (cat. \# sc-31638) anti-HSC70 (cat. \#sc-7298), and anti-LAMP1 1D4B (cat. \# sc-19992) were from Santa Cruz Biotechnology; anti-EHD1 (cat. \# ab109311) was from Abcam; anti-ß-Actin (cat. \# A5316) was from Sigma-Aldrich; anti-pErk42/44 (cat. \# 9101) and P-M-CSF Receptor (Y723, 49C10) was from Cell Signaling Technology; F4/80-APC, BM8 (cat. \# 17-4801-82), anti-GM130 (cat. \# 610822), CD16/32 (cat. \# 14-0161), and APCconjugated anti-Annexin V (cat. \# 17-8007-72) were from eBiosciences; a polyclonal rabbit 
antibody recognizing EHD1 and EHD4 was generated in-house was described previously (45). Secondary fluorochrome-conjugated antibodies were from Life Technologies.

\subsection{Mice Generation \& Genotyping}

Ehd1 $1^{f l f l}$ mice in a predominantly C57BL/6 background [37,38] were crossed with tamoxifen-inducible CreERT2 expressing mice from Jackson Laboratories

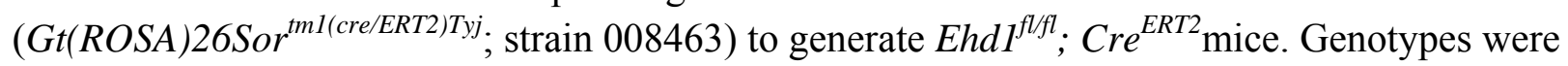
confirmed by subjecting tail clip DNA to PCR analysis using the KAPA mouse genotyping kit (KAPA Biosystems) and primers pairs described previously [36,38]. Mice were treated humanely according to the National Institutes of Health (NIH) and The University of Nebraska Medical Center guidelines. Animal studies were pre-approved by the Institutional Animal Care and Use Committee (\#07-061-FC12).

\subsection{Bone marrow derived macrophage culture (BMDMs)}

Bonemarrow was harvested from 8-12 week old mice by removing the femur bones, cutting the ends, and flushing with ice-cold PBS. Red blood cells where lysed with solute-free $\mathrm{H}_{2} \mathrm{O}$ and the bone marrow was plated on tissue culture coated dishes for 16 hours to allow mature/non-progenitor cells to attach. The non-adherent progenitor population was collected by pipetting and re-plated in 15-cm petri dishes (non-tissue culture treated). Cells were then incubated for 6 days in BMDM media (RPMI w/ 15\% FBS, 10\% L929 supernatant [39] or 30 $\mathrm{ng} / \mathrm{ml}$ recombinant CSF-1 and $1 \%$ penicillin/streptomycin) at $37^{\circ} \mathrm{C}$ and $5 \% \mathrm{CO}_{2}$, with change of medium every 2 days. To assess the impact of EHD1 deletion, Ehd1 ${ }^{f l / l}$; Cre ${ }^{E R T 2}$ BMDMs were either cultured in regular medium (control/wildtype EHD1 expression) or medium containing $200 \mathrm{nM}$ 4-hydroxytamoxifen (TAM) for 4 days to induce EHD1 deletion. This protocol was based on initial time course and titration studies to determine optimal culture conditions.

\subsection{Fluorescence Activated Cell Sorting (FACS)}

BMDMs were washed with ice-cold PBS and blocked for 10 minutes for non-specific binding with CD16/32 (Fc Blocker) and then incubated with appropriate antibodies at a dilution of 1:400 and put in the dark and on ice for 30min. Cells were then pelleted, washed with PBS twice, and suspended in $400 \mu \mathrm{l}$ of $0.1 \% \mathrm{BSA} / \mathrm{PBS}$, put on ice, and protected from light.

\subsection{Quantitative real-time-PCR ( $q R T-P C R)$}

RNA was isolated from BMDMs grown on $10-\mathrm{cm}$ tissue culture plates using Trizol reagent, and 1 ug of total RNA was reverse transcribed using the QuantiTect Reverse Transcription Kit (QAIGEN). 10\% volume of the RT reaction of the ensuing cDNA was used 
with QuantiTect SYBR Green qRT-PCR Kit (QAIGEN) for quantitative qRT-PCR of mouse CSF-1R using the primer sequences (5'-3'): GCAGTACCACCATCCACTTGTA;

GTGAGACACTGTCCTTCAGTGC. GAPDH was used as a control, and changes in CSF-1R were calculated using the cycle threshold method [40]. Each reaction was performed in triplicate in a volume of $50 \mathrm{uL}$ with primers at a final concentration of $250 \mathrm{nM}$.

2.6 ${ }^{3}$ H-thymidine incorporation assay

BMDMs were deprived of CSF-1 for 16 hours and plated in 48-well tissue culture plates at $1 \times 10^{4}$ cells/well and allowed to attach overnight at $37^{\circ} \mathrm{C}, 5 \% \mathrm{CO}$. Cells were then treated with $30 \mathrm{ng} / \mathrm{ml} \mathrm{CSF-1} \mathrm{and} \mathrm{allowed} \mathrm{to} \mathrm{proliferate} \mathrm{for} \mathrm{2,} 4$ or 6 days. Cells were pulsed with $4 \mu \mathrm{Ci} /$ well of ${ }^{3} \mathrm{H}$-Thymidine for the last 6 hours of the assay. Radioactivity incorporated into DNA was collected by washing the cells with $10 \%$ TCA per well followed by $300 \mu 1$ per well 0.2 $\mathrm{N} \mathrm{NaOH}$ at room temperature to dissolve DNA. Each sample was transferred into vials with 5 $\mathrm{mL}$ of scintillation fluid and counts per minute (c.p.m.) were recorded with a scintillation counter.

\subsection{CFSE dye dilution assay}

Macrophages were cultured with recombinant CSF-1 $(30 \mathrm{ng} / \mathrm{mL})$ for 2, 4 or 6 days after initial CFSE population staining (Day 0) according to ThermoFisher Scientific's protocol. Dilution of CFSE fluorescence as an indicator of cell division was assessed viaFluorescence Activated Cell Sorting(FACS) analysis The CFSE geometric mean fluorescence intensity (MFI)was used to access the degree of CSF1-induced cell division.

\subsection{Microscopy \& Imaging}

Imaging using immunofluorescence was performed as previously described [41] with minor modifications. BMDMs were grown on glass coverslips and fixed with $2 \%$ PFA/PBS for 5 min. To assess the intracellular pools of CSF-1R, the cells were fixed and permeabilized in methanol for 10 minutes at $-20^{\circ} \mathrm{C}$. The cells were then blocked with 1:400 CD16/32 (Fc Blocker) in $1 \% \mathrm{BSA} / \mathrm{PBS}$ for 30 minutes and incubated with primary antibodies in $1 \% \mathrm{BSA} / \mathrm{PBS}$ overnight. After 3 washes in PBS, the cells were incubated with the appropriate fluorochromeconjugated secondary antibody for 45 minutes at room temperature, washed and mounted using Fluoromount-G (Southern Biotech, Cat. \# 0100-01) or VECTASHEILD mounting medium (Vector laboratories, Cat. \# H-1400 and H-1500). Images were acquired using a LeicaCTR4000 inverted microscope equipped with an QICAM 12-bit color camera, 12V $100 \mathrm{~W}$ halogen lamp, QCapture software and 60x oil immersion lens (For CSF-1R) and a Zeiss 710 Meta Confocal Laser Scanning microscope (Carl Zeiss) using a $63 \times$ objective with a numerical aperture of 1.0 
and appropriate filters. Merged fluorescence pictures were generated and analyzed using ZEN® 2012 software from Carl Zeiss.

\subsection{Macrophage spreading assay}

BMDMs cultured without(Control) or with (EHD1-KO) TAM were plated on coverslips and allowed to attach for 2 days. The cells were then deprived of CSF-1 for 16 hours and subsequently stimulated with $100 \mathrm{ng} / \mathrm{mL} \mathrm{CSF}-1$ for 10 minutes. Cells were fixed for 10 minutes with 4\% PFA, washed with PBS 3 washes for 10 minutes, and stained using conjugated Alexa Fluor ${ }^{\circledR} 594$ Phalloidin (ThermoFisher Scientific). ImageJ software was used to quantify the surface area of macrophages.

\subsection{Macrophage trans-wellmigration assay}

BMDMs were deprived of CSF-1 for 4hours and placed in the upper chambers of $5 \mu \mathrm{m}$ pore inserts (Corning, cat. \# 3421) at $1 \times 10^{5}$ cells per chamber in $100 \mu 1$ RPMI without CSF-1. Cells were then allowed to migrate towards CSF-1 in the lower chamber $(30 \mathrm{ng} / \mathrm{ml}$ ) for 3 hours at $37^{\circ} \mathrm{C}$. Cells on the lower surface of membranes were fixed with $100 \%$ methanoland stained with HEMAfor5 minutes. Cells were visualized under abright field microscope. Migrated cells were counted in 10 fields per insert and the total number of migrated cells was calculated for control and EHD1-KO BMDMs.

\subsection{Western blotting}

Western blotting was performed as described [38]. Briefly, BMDMs were washed with ice-cold PBS and lysed in ice-cold Triton X-100 lysis buffer $(0.5 \%$ Triton X-100, $50 \mathrm{mM}$ Tris pH 7.5, $150 \mathrm{mM}$ sodium chloride, and EDTA-free protease inhibitor cocktail). The lysates were vortexed, centrifuged at 13,000 rpm for 30 minutes at $4^{\circ} \mathrm{C}$, and supernatants collected. Protein quantification was done using the Bicinconic acid (BCA) assay $40 \mu \mathrm{g}$ of lysate protein per sample was resolved by SDS/PAGE and transferred to a PVDF membrane. The membranes were blocked in TBS/1\% Bovine Serum Albumin (BSA), incubated with the appropriate primary antibodies diluted in TBS- $0.1 \%$ tween 20 overnight at $4^{\circ} \mathrm{C}$ and washed in TBS- $0.1 \%$ tween $(3 \mathrm{x}$ for 10 minutes) followed by a 1-hour incubation with HRP-conjugated secondary antibody at room temperature. The membrane was then washed in TBS- $0.1 \%$ tween ( $3 \mathrm{x}$ for 10 minutes each) and ECL-based detection performed.

\section{$2.12\left[{ }^{35} \mathrm{~S}\right]$-methionine/cysteine metabolic labeling and Immunoprecipitation CSF-1R}

BMDMs were seeded at $5 \times 10^{6}$ cells $/ 10-\mathrm{cm}$ plate in CSF-1 containing medium and cultured for 16 hours. The cells were washed and incubated in methionine/cysteine-free RMPI- 
1640 medium supplemented with $15 \% \mathrm{FBS}$ and $30 \mathrm{ng} / \mathrm{mL}$ recombinant CSF-1 for 30 minutes at $37^{\circ} \mathrm{C}$ and pulsed with $0.1 \mathrm{mCi}$ of $\left[{ }^{35} \mathrm{~S}\right]$ for 15 minutes at $37^{\circ} \mathrm{C}$. Cells were then washed and incubated with regular culture medium containing a 20 -fold excess of unlabeled methionine/cysteine for $0,15,30$, and 60 minutes of chase. Cells were rinsed with ice-cold PBS and lysed in ice-cold Triton X-100 lysis buffer with EDTA-free protease inhibitor cocktail. AntiCSF-1R immunoprecipitations were carried out using $500 \mu \mathrm{g}$ samples of cleared lysate protein and anti-CSF-1Rantibody followed by capture with Protein-A Sepharose beads. Samples were resolved by SDS/PAGE ( $8 \%$ gel). Gels were fixed, dried, and incubated with Auto-Fluor to amplify $\left[{ }^{35} \mathrm{~S}\right]$ signals and autoradiography was performed at $-80^{\circ} \mathrm{C}$ in metal cassettes with intensifying screens.

\subsection{Statistics}

Unpaired student's $t$ tests were used to calculate $p$-values. Data is presented as mean \pm SEM, $\mathrm{p}<0.05$ defines thethreshold of statistical significance.

\section{Results}

\subsection{EHD1-KO diminishes CSF-1R signalling in BMDMs}

To determine if deletion of EHD1 had a functional impact in BMDMs, we first assessed whether or not EHD1-KO bone marrow progenitors maintained the ability to differentiate into mature macrophages (i.e. F4/80 ${ }^{+}$). EHD1 $1^{f l f l}$; CreERT2 BMDMs grown in the absence or presence of TAM were stained with F4/80 antibody and analyzed with FACS. Greater than $99 \%$ of both (-) and (+) TAM treated populations stained positively for F4/80 (Suppl. Fig. 1A), demonstrating the ability of these cells to differentiate into mature macrophage. We nextperformed toxicity controls to verify that any phenotypic effects observed intamoxifeninduced EHD1-KO BMDM was a bona fide effect of EHD1 deletion and not an effect of tamoxifen treatment (Suppl. Fig. 1B-D). These assays showed tamoxifen did not attenuate CSF1induced CSF-1R signalling responses in BMDMs. To assess the function of EHD1 in macrophage CSF1-induced cellular responses, we assayed the effects of CSF1-induced proliferation oncontrol and EHD-KO BMDMs using two independent methods: ${ }^{3} \mathrm{H}$-thymidine incorporation and CFSE dye dilution assays. These two assays have been previously used to assess CSF1-induced macrophage proliferation [54, 55]. Both assays showed EHD1-KO macrophages had significantly reduced proliferative responses when stimulated with CSF-1 (Fig. 1A-C).

We next sought to further assess the effects of EHD1-KO on CSF-1R ligand-induced activation,signalling, andactivation of the MAPK pathway - awell-known driver of proliferation $[44,45]$. We noted the total CSF-1R levels before and after stimulation were lower in 
CSF1-deprived EHD1-KO vs. control BMDMs (Fig. 1D and 1F; 0 time points). Commensurate with diminishedtotal macrophage CSF-1R, accumulation of pERK(Fig. 1D-E). and pCSF1R(Suppl. Fig. 2A-B) in EHD1-KO BMDMs was significantly lower than in control BMDMs.

\subsection{EHD1-KO BMDMs have decreased spreading and migration}

As stimulation through CSF-1R is known to correlate with rapid macrophage spreading [46], we next examined the impact of EHD1 deletion on this cellular response.BMDMs grown without (Control) or with (EHD1-KO) TAM were deprived of CSF-1 for 16 hours and then were stimulated with CSF-1 for 10 minutes, fixed, and stained for actin to assess the extent of cell spreading. EHD1-KO BMDMs exhibited reduced spreading in response to CSF-1 stimulation (Fig. 2A-B).We next assessed the impact of EHD1 deletion on CSF1-induced trans-well migration, a well-established response to CSF-1R stimulation [47]. Compared to control, EHD1KOBMDMs showed a significant reduction in migration (Fig. 2C-D). These data suggest EHD1 positively regulates macrophage spreading and migrationthrough modulation of CSF1Rsignalling.

\subsection{Expression of cell surface CSF-1R is reduced in EHD1-deficient macrophages}

Given the deficits in macrophage functional responses to CSF-1R stimulation (i.e., proliferation, spreading, and migration), we next sought to investigate the expression of CSF-1R at the cell surface in Control and EHD1-KO BMDMs. AsBMDMs require the continuous presence of CSF-1 in vitro, surface levels of CSF-1R are low under steady state conditions due to continuous receptor activation and lysosomal trafficking of surface CSF-1R [15,24]. Therefore, we assessed the cell surface CSF-1R levels by flow cytometry after 2, 4, 8 or 16 hours of ligand deprivation from the culture medium (CSF-1deprivation) to allow newly synthesized CSF-1R to accumulate at the cell surface. Initially low CSF-1R surface levels increased steadily over time in ControlBMDMs, however cell surface CSF-1R in EHD1-KO BMDMs accumulated at a significantly lower rate. Surface expression of CSF-1R was significantly less at all-time points following CSF-1 deprivation, compared to control cells (Fig. 3A-B). Thus, loss of EHD1 leads to reduced CSF-1R present at the cell surface in BMDMs available for activation by CSF-1.

\subsection{Total CSF-1R expression is reduced in EHD1-KO BMDMs}

To investigate the mechanism responsible for reduced cell surface expression of CSF-1R on EHD1-KO BMDMs, we first examined the accumulation of total cellular CSF-1R protein after CSF-1was removed from the culture medium using western blotting. Ehd $1^{\text {fl/fl }}$; Cre ${ }^{E R T 2}$ BMDMs grown in CSF1-containing medium without (Control) or with (EHD1-KO) TAM were switched to medium without CSF-1 for 2, 4, 8 or 16 hours, and cellular lysates were analyzed by immunoblotting for CSF-1R. EHD1-KO BMDMs showed a significant and 
substantial reduction in the total CSF-1R protein levels at each time point (Fig. 3C-D). These findings indicate that total CSF-1R protein levels are dependent on the presence of EHD1 in the cell.

\subsection{EHD1 is required for the display of CSF-1R on the macrophage cell surface}

Given the reduction in surface and total CSF-1R in EHD1-KO BMDMs, we carried out qRT-PCR analysis to assess the levels of CSF-1R mRNA expression in control and EHD1-KO BMDMs. Ehd1 ${ }^{\text {fl/fl }}$ Cre ${ }^{E R T 2}$ BMDMs cultured with (Control) or without (EHD1-KO) TAM were harvested under steady state culture conditions or after 16 hours CSF-1 deprivation. CSF-1R mRNA levels increased nearly 50\% in CSF-1 deprived control BMDMs compared to control BMDMs cultured under steady-state conditions. Likewise, CSF-1R mRNA levels in CSF-1 deprived EHD1-KO BMDMs increased in a similar pattern to control BMDMs and no significant difference in CSF-1R mRNA expression was observed between control and EHD1KO BMDMs under steady state or ligand deprived conditions (Fig. 4A). These data demonstrate that the reduction in surface and total CSF-1R levels in EHD1-KO BMDMs is not due to decreased CSF-1R mRNA expression.

In order to assess if EHD1 deletion affects CSF-1R protein synthesis and/or its posttranslational maturation and transport to the cell surface, we preformed metabolic pulse-labeling of the receptor with $\left[{ }^{35} \mathrm{~S}\right]$-methionine/cysteine followed by chase in CSF1-containing culture medium. Under these conditions, newly synthesized and mature (i.e., glycosylated) CSF-1R that reaches the cell surface is expected to bind to CSF-1and be targeted for degradation in the lysosome, resulting in a reduction in radioactive signals of mature CSF-1R protein at later time points of chase. Equal quantities of lysates collected at various time points were subjected to anti-CSF-1R immunoprecipitation followed by autoradiography to visualize the immature and mature CSF-1R polypeptides. The signals of immature CSF-1R synthesized during pulselabeling (time 0) were comparable between control and EHD1-KO BMDMs (Fig. 4B; lower band), thus excluding any alteration in the rate of protein synthesis as the determinant of reduced total CSF-1R levels in EHD1-KO cells observed by Western blot (Fig. 3C-D). Analysis at various times during chase showed that the conversion of immature CSF-1R to a fully glycosylated protein at agreater molecular weight [12] was similar in control and EHD1-KO BMDMs with the majority of immature protein being converting to the mature form by $30 \mathrm{~min}$ of chase (Fig. 4B). Notably, mature CSF-1R signals in control BMDMs were clearly diminished by 60 min of chase, reflecting the receptor's arrival at the cell surface and subsequent CSF1induced internalization and degradation (Fig. 4B) [21]. In contrast, the mature CSF-1R signals remained prominent, and nearly unaltered, at 60 min of chase in EHD1-KO BMDMs (Fig. 4B), indicating a block in CSF-1R receptor trafficking occurs after glycosylation in the Golgi but before ligand-induced internalization and lysosomal degradation. Given the observation that display of CSF-1R at the cell surface is decreased, these data support the conclusion that in EHD1-KO BMDMs the block in CSF-1R receptor trafficking occurs during the stage of receptor 
transport from the Golgi to the cell surface. In summary, these findings indicate that transcription, translation, and post-translational modification of CSF-1R are unaltered in EHD1$\mathrm{KO}$ macrophages andthat EHD1 is required for the transport of CSF-1R to the cell surface after receptor glycosylation in the Golgi.

\subsection{A pool of CSF-1R localized to a $E H D 1^{+} / G M 130^{+}$compartment}

Given the results of pulse-chase analyses, we asked if EHD1 co-localizes with CSF-1R at the Golgi apparatus. Three-color confocal imaging was performed on fixed and permeabilized control and EHD-KO BMDMs deprived of CSF-1 for 2, 4, 8, and 16 hours to allow for CSF-1R synthesis and accumulation. In control BMDMs, EHD1 strongly co-localized with the Golgi marker GM130and a pool of CSF-1R localized to this EHD $1^{+} / \mathrm{GM} 130^{+}$compartment (Fig. 5). No EHD1 staining was detected in TAM-induced EHD1-KO BMDMs (Fig. 5). EHD1-KO BMDMs also exhibited a pool of CSF-1R localized to the GM130 ${ }^{+}$Golgi compartment, with diminished CSF-1R signals near the cell surface (Fig 5). Together with the results of the metabolic pulsechase (Fig. 4B), these data support the conclusion that EHD1 is involved in directing Golgilocalized CSF-1R to the cell surface, and that in EHD1-KO BMDMs CSF-1R may transit to a different, potentially degradative, cellular compartment.

\subsection{CSF-1Ris shunted to the lysosome in EHD1-KO BMDMs and results in receptor degradation}

To investigate CSF-1R degradation, Ehd $1^{\text {fl/fl }}$; $C r e^{E R T 2}$ BMDMs cultured in the absence (control) or presence (EHD1-KO) of TAM were deprived of CSF-1 for 4 hours either in the absence or presence of lysosomal proton pump blocker Bafilomycin A1 (Baf-A1). Western blot analysis demonstrated lower accumulated levels of total CSF-1R protein in EHD1-KO macrophages compared to control when cultured in the absence of Baf-A1 (Fig. 6A-B; $1^{\text {st }}$ and $3^{\text {rd }}$ lanes and Fig. 3C-D). In contrast, inclusion of Baf-A1 led to a dramatic and statistically significant increase in CSF-1R levels in EHD1-KO BMDMswhen compared withEHD1-KO BMDMs cultured in the absence of Baf-A1, and nearly approached the levels in control+Baf-A1 BMDMs (Fig. 6A-B). These results suggest that CSF-1R rapidly transits from the Golgi to the lysosome in EHD1-KO BMDMs and results in receptor degradation (Fig. 6A-B).Moreover, increased degradation of CSF-1R (without change in CSF-1R synthesis) in EHD1-KO BMDMs provides amechanistic explanationfor our findings that EHD1-KO macrophages have decreased CSF-1R surface and total levels.

To further demonstrate that CSF-1R is shunted to the lysosome, we carried out confocal imaging of control vs. EHD1-KO BMDMs deprived of CSF-1 for 4 hours in the presence of BafA1 and stained for CSF-1R and the lysosomal marker LAMP1. In contrast to a small pool of CSF-1R that localized in LAMP $1^{+}$lysosomes in control BMDMs, a significantly larger pool of CSF-1R co-localized to LAMP1 ${ }^{+}$lysosomes in EHD1-KO BMDMs (Fig. 6C-D). Overall, these results support the role of EHD1 in the transport of newly synthesizedCSF-1R from the Golgi to 
the macrophage cell surface. Collectively, these data suggest EHD1 governs CSF-1R signalling via control of CSF-1R presentation on the macrophage cell surface.

\section{Discussion}

CSF-1R is essential for the development and varied functions of monocyte-macrophage lineage cells [2]. Abnormal CSF-1R-dependent macrophage functions contribute to variety of human diseases and aberrant CSF-1R signalling is a contributor to oncogenesis $[3,11,48,49]$. A pre-requisite for all CSF-1R-mediated macrophage biological functions is the display of the receptor at the cell surface in order for ligand to bind and initiate signal transduction cascades. Mechanisms that govern the post-synthesis/maturation traffic of CSF-1R to the cell surface are therefore biologically pivotal, yet nothing is known about these mechanisms at the present time. Here, we used primary bone marrow-derived macrophages (BMDMs) derived from an inducible knockout mouse model to provide evidence that EHD1, a member of the EHD family of endocytic recycling regulators, serves a novel and critical role in ensuring Golgi to cell surface traffic of CSF-1R. We show that this novel function of EHD1 in delivering CSF-1R from the Golgi to the membrane. In the absence of EHD1, CSF-1R is shunted to the lysosome. Our studies show that EHD1-dependent cell surface transport of CSF-1R ensures subsequent ligandinduced cellular activation. Our findings that EHD1 functions as a novel regulator CSF-1R transport to the cell surface help assign a new functional role to EHD1.

As much of the functional biology of EHD proteins has emerged in the context of nonsignalling receptors, we envisioned a potential role of EHD1 in the traffic of the RTK CSF-1R in the absence of its ligand induced trafficking. CSF-1R binding to CSF-1 has been established to induce rapid internalization and lysosomal degradation [16] of the activated receptor, such that surface and total levels of CSF-1R in BMDMs cultured in the continual presence of CSF-1 (steady state) are relatively low. By deliberately examining the levels of surface and total CSF1R after switching BMDMs from steady-state to CSF-1deprived culture medium, our studies directly focused on the accumulation and traffic of newly synthesized CSF-1R. Analyses of the cell surface and total CSF-1R protein under these conditions, using a combination of flow cytometry (Fig. 3A-B) western blotting (Fig. 3C-D), metabolic pulse-chase (Fig. 4B) and immunofluorescence imaging of non-permeabilized(Suppl. Fig. 3A-B) or permeabilized (Suppl. Fig. 3C-D) BMDMs demonstrated a clear impairment in the ability of newly synthesized CSF$1 \mathrm{R}$ to be transported to the cell surface and an overall reduction in CSF-1R levels when EHD1 was deleted.

Previous studies have shown that reduced surface expression of CSF-1R can result from defective glycosylation. This is seen upon expression of the HIV protein NEF, which inhibits glycosylation through an incompletely understood mechanism involving NEF activation of the SRC-family tyrosine kinase HCK as well as through an HCK-independent mechanism [50,51]. Naturally-occurring loss-of-function mutations of CSF-1R have been identified in patients with 
hereditary diffuse leukoencephalopathy with spheroids (HDLS), and most of these mutations result in reduced CSF-1R protein expression [8]. In both of the aforementioned cases, CSF-1R glycosylation in the Golgi is defective. In contrast, our metabolic pulse-chase studies (Fig. 4B) showed that immature CSF-1R was fully converted into a mature form (fully glycosylated with higher molecular weight) in EHD1-KO BMDMs with essentially the same kinetics as in control cells. These findings clearly pointed to the possibility that post-synthetic transport of CSF-1R after its Golgi processing was defective in EHD1-KO BMDMs. Consistent with this idea, we observed that CSF-1R co-localizes Golgi markerGM130 (Fig. 5). Our metabolic pulse-chase studies carried out in the presence of CSF-1 exposed the newly synthesized CSF-1R to its ligand, which promoted its rapid degradation. While this was clearly seen in control BMDMs, the mature band of CSF-1R remained intact for the duration of the experiment in EHD1-KO BMDMs (Fig. 4B), supporting the conclusion that ${ }^{35}$ S-labled CSF-1R did not reach the cell surface, consistent with results from flow cytometry (Fig. 3A-B) and immunofluorescencemicroscopy (Suppl. Fig. 3A-B) analyses. When combined with the observed reduction in total CSF-1R levels in EHD1-KO BMDMs, these results led us to hypothesize that lack of EHD1 allows CSF-1R traffic from the Golgi to a compartment where it is degraded. As RTKs are known to be degraded in lysosomes [15,22] and a variety of receptors are known to traffic from the Golgi to the lysosome directly [52,53], we considered the possibility that in EHD1-KO BMDMs CSF-1R is alternatively sorted to the lysosome and subsequently degraded. Our Western blotting analyses demonstrating that CSF-1R protein accumulates in EHD1-KO BMDMs when cultured in the presence of Baf-A1 (Fig. 6A-B), together with confocal imaging of Baf-A1 treated BMDMs (Fig. 6C-D) support this mechanism.

It is of obvious interest to assess if the EHD1-dependent CSF-1R transport pathway we describe is also important for other RTKs or represents a more restricted adaptation for CSF-1R. How the balance of these pathway operates and whether it is tunable during development, differentiation or functional responses of macrophage-lineage or other cells will be of great interest, especially in the context of human diseases in which inhibition of CSF-1R signalling would be desirable $[3,9,54,55]$.

\section{Conclusions}

In summary, our data suggestthat exit of fully glycosylated and mature CSF-1R from the Golgi is a regulated process under the control of two competing sorting processes: a dominant EHD1-dependent pathway to facilitate traffic to the cell surface and an alternate pathway of traffic to lysosomes for degradation. Our findings support the conclusion that EHD1-dependent CSF-1R sorting decisions at the Golgi are of key significance in determining the ultimate level of CSF-1R signalling and downstream biological responses. 


\section{Acknowledgments}

This work was supported by: the NIH grants CA105489, CA87986, CA99163 and CA116552 to HB, CA105489-supplement to FI and CA96844 and CA144027 to VB;

Department of Defense grants W81XWH-07-1-0351 and W81XWH-11-1-0171 (VB); the NE DHHS LB-506 (2014-01) and LB606 (18123-Y3) grants (HB); Institutional Development Award (IDeA) from the NIGMS of the NIH under grant number P30 GM106397. The UNMC Confocal, Flow Cytometry and other Core facilities from the NCI Cancer Center Support Grant (P30CA036727) to Fred \& Pamela Buffett Cancer Center and the Nebraska Research Initiative.

LC and TB are trainees under the NCI Cancer Biology Training Grant (T32CA009476). WA was a recipient of a UNMC graduate fellowship.

We thank Garland (Michael) Upchurch for insight, suggestions, and critical reading/proofing of the manuscript. 


\section{References}

[1] S.J. Jenkins, D.A. Hume, Homeostasis in the mononuclear phagocyte system, Trends Immunol. 35 (2014) 358-367. doi:10.1016/j.it.2014.06.006.

[2] D.A. Hume, Differentiation and heterogeneity in the mononuclear phagocyte system, Mucosal Immunol. 1 (2008) 432-441. doi:10.1038/mi.2008.36.

[3] V. Chitu, E.R. Stanley, Colony-stimulating factor-1 in immunity and inflammation, Curr. Opin. Immunol. 18 (2006) 39-48. doi:10.1016/j.coi.2005.11.006.

[4] J.A. Hamilton, Colony-stimulating factors in inflammation and autoimmunity John, Nat. Immunol. 23 (2008) 533-544. doi:10.1016/S1471-4906(02)02260-3.

[5] J.W. Pollard, Trophic macrophages in development and disease., Nat. Rev. Immunol. 9 (2009) 259-70. doi:10.1038/nri2528.

[6] M.L. Toh, J.Y. Bonnefoy, N. Accart, S. Cochin, S. Pohle, H. Haegel, et al., Bone- and cartilage-protective effects of a monoclonal antibody against colony-stimulating factor 1 receptor in experimental arthritis, Arthritis Rheumatol. 66 (2014) 2989-3000. doi:10.1002/art.38624.

[7] J.F.J. Bogie, P. Stinissen, J.J.A. Hendriks, Macrophage subsets and microglia in multiple sclerosis, Acta Neuropathol. 128 (2014) 191-213. doi:10.1007/s00401-014-1310-2.

[8] C. Sundal, S. Fujioka, J.A. Van Gerpen, C. Wider, A.M. Nicholson, M. Baker, et al., Parkinsonian features in hereditary diffuse leukoencephalopathy with spheroids (HDLS) and CSF1R mutations, Park. Relat. Disord. 19 (2013) 869-877. doi:10.1016/j.parkreldis.2013.05.013.

[9] Y. Aikawa, T. Katsumoto, P. Zhang, H. Shima, M. Shino, K. Terui, et al., PU.1-mediated upregulation of CSF1R is crucial for leukemia stem cell potential induced by MOZ-TIF2., Nat. Med. 16 (2010) 580-585, 1p following 585. doi:10.1038/nm.2122.

[10] B.-Z. Qian, J. Li, H. Zhang, T. Kitamura, J. Zhang, L.R. Campion, et al., CCL2 recruits inflammatory monocytes to facilitate breast-tumour metastasis., Nature. 475 (2011) 222 5. doi:10.1038/nature10138.

[11] D. Laoui, E. van Overmeire, P. de Baetselier, J.A. van Ginderachter, G. Raes, Functional relationship between tumor-associated macrophages and macrophage colony-stimulating factor as contributors to cancer progression, Front. Immunol. 5 (2014) 1-15. doi:10.3389/fimmu.2014.00489.

[12] F.J. Pixley, E.R. Stanley, CSF-1 regulation of the wandering macrophage: Complexity in action, Trends Cell Biol. 14 (2004) 628-638. doi:10.1016/j.tcb.2004.09.016.

[13] S. Wei, S. Nandi, V. Chitu, Y.-G.Y.-G. Yeung, W. Yu, M. Huang, et al., Functional overlap but differential expression of CSF-1 and IL-34 in their CSF-1 receptor-mediated regulation of myeloid cells, J. Leukoc. Biol. 88 (2010) 495-505. doi:10.1189/jlb.1209822.

[14] H. Liu, C. Leo, X. Chen, B.R. Wong, L.T. Williams, H. Lin, et al., The mechanism of shared but distinct CSF-1R signaling by the non-homologous cytokines IL-34 and CSF-1, Biochim. Biophys. Acta - Proteins Proteomics. 1824 (2012) 938-945. 
doi:10.1016/j.bbapap.2012.04.012.

[15] P.S.W. Lee, Y. Wang, M.G. Dominguez, Y.G. Yeung, M.A. Murphy, D.D.L. Bowtell, et al., The Cbl protooncoprotein stimulates CSF-1 receptor multiubiquitination and endocytosis, and attenuates macrophage proliferation, EMBO J. 18 (1999) 3616-3628. doi:10.1093/emboj/18.13.3616.

[16] Y. Wang, Y.G. Yeung, W.Y. Langdon, E.R. Stanley, c-Cbl is transiently tyrosinephosphorylated, ubiquitinated, and membrane-targeted following CSF-1 stimulation of macrophages, J. Biol. Chem. 271 (1996) 17-20. doi:10.1074/jbc.271.1.17.

[17] Y. Xiong, D. Song, Y. Cai, W. Yu, Y.G. Yeung, E.R. Stanley, A CSF-1 receptor phosphotyrosine 559 signaling pathway regulates receptor ubiquitination and tyrosine phosphorylation, J. Biol. Chem. 286 (2011) 952-960. doi:10.1074/jbc.M110.166702.

[18] B. Mohapatra, G. Ahmad, S. Nadeau, N. Zutshi, W. An, S. Scheffe, et al., Protein tyrosine kinase regulation by ubiquitination: Critical roles of Cbl-family ubiquitin ligases, Biochim. Biophys. Acta. 1833 (2013) 122-139. doi:10.1016/j.bbamcr.2012.10.010.

[19] G. Ahmad, B.C. Mohapatra, N.A. Schulte, S.A. Nadeau, H. Luan, N. Zutshi, et al., Cblfamily ubiquitin ligases and their recruitment of CIN85 are largely dispensable for epidermal growth factor receptor endocytosis, Int. J. Biochem. Cell Biol. 57 (2014) 123134. doi:10.1016/j.biocel.2014.10.019.

[20] S. Miyake, M.L. Lupher, B. Druker, H. Band, The tyrosine kinase regulator Cbl enhances the ubiquitination and degradation of the platelet-derived growth factor receptor alpha., Proc. Natl. Acad. Sci. U. S. A. 95 (1998) 7927-32. doi:10.1073/pnas.95.14.7927.

[21] Y. Wang, Y.G. Yeung, E.R. Stanley, CSF-1 stimulated multiubiquitination of the CSF-1 receptor and of $\mathrm{Cbl}$ follows their tyrosine phosphorylation and association with other signaling proteins, J. Cell. Biochem. 72 (1999) 119-134. doi:10.1002/(SICI)10974644(19990101)72:1<119::AID-JCB13>3.0.CO;2-R.

[22] L.L.K. Goh, A. Sorkin, Endocytosis of receptor tyrosine kinases, Cold Spring Harb. Perspect. ... 5 (2013) a017459. doi:10.1101/cshperspect.a017459.

[23] J. Huynh, M.Q. Kwa, A.D. Cook, J.A. Hamilton, G.M. Scholz, CSF-1 receptor signalling from endosomes mediates the sustained activation of Erk1/2 and Akt in macrophages, Cell. Signal. 24 (2012) 1753-1761. doi:10.1016/j.cellsig.2012.04.022.

[24] L.J. Guilbert, E.R. Stanley, The interaction of 125I-colony-stimulating factor-1 with bone marrow-derived macrophages, J. Biol. Chem. 261 (1986) 4024-4032. http://www.ncbi.nlm.nih.gov/pubmed/3485098 (accessed June 19, 2015).

[25] N. Naslavsky, S. Caplan, EHD proteins: key conductors of endocytic transport, Trends Cell Biol. 21 (2010) 122-131. doi:10.1016/j.tcb.2010.10.003.

[26] B.D. Grant, S. Caplan, Mechanisms of EHD/RME-1 protein function in endocytic transport, Traffic. 9 (2008) 2043-2052. doi:10.1111/j.1600-0854.2008.00834.x.

[27] O. Daumke, R. Lundmark, Y. Vallis, S. Martens, P.J.G. Butler, H.T. McMahon, Architectural and mechanistic insights into an EHD ATPase involved in membrane remodelling., Nature. 449 (2007) 923-7. doi:10.1038/nature06173. 
[28] A.D. Posey, P. Pytel, K. Gardikiotes, A.R. Demonbreun, M. Rainey, M. George, et al., Endocytic recycling proteins EHD1 and EHD2 interact with Fer-1-like-5 (Fer1L5) and mediate myoblast fusion, J. Biol. Chem. 286 (2011) 7379-7388.

doi:10.1074/jbc.M110.157222.

[29] A.D. Posey, K.E. Swanson, M.G. Alvarez, S. Krishnan, J.U. Earley, H. Band, et al., EHD1 mediates vesicle trafficking required for normal muscle growth and transverse tubule development, Dev. Biol. 387 (2014) 179-190. doi:10.1016/j.ydbio.2014.01.004.

[30] M. George, M.A. Rainey, M. Naramura, K.W. Foster, M.S. Holzapfel, L.L. Willoughby, et al., Renal thrombotic microangiopathy in mice with combined deletion of endocytic recycling regulators EHD3 and EHD4, PLoS One. 6 (2011) e17838.

doi:10.1371/journal.pone.0017838.

[31] J. Curran, M.A. Makara, S.C. Little, H. Musa, B. Liu, X. Wu, et al., EHD3-dependent endosome pathway regulates cardiac membrane excitability and physiology, Circ. Res. 115 (2014) 68-78. doi:10.1161/CIRCRESAHA.115.304149.

[32] J. Curran, H. Musa, C.F. Kline, M.A. Makara, S.C. Little, J.D. Higgins, et al., Eps 15 homology domain-containing protein 3 regulates cardiac T-type $\mathrm{Ca}^{2+}$ channel targeting and function in the atria, J. Biol. Chem. 290 (2015) 12210-12221.

doi:10.1074/jbc.M115.646893.

[33] H. Gudmundsson, J. Curran, F. Kashef, J.S. Snyder, S.A. Smith, P. Vargas-Pinto, et al., Differential regulation of EHD3 in human and mammalian heart failure, J. Mol. Cell. Cardiol. 52 (2012) 1183-1190. doi:10.1016/j.yjmcc.2012.02.008.

[34] S. Sengupta, M. George, K.K. Miller, K. Naik, J. Chou, M.A. Cheatham, et al., EHD4 and CDH23 are interacting partners in cochlear hair cells, J. Biol. Chem. 284 (2009) 2012120129. doi:10.1074/jbc.M109.025668.

[35] M. George, M.A. Rainey, M. Naramura, G. Ying, D.W. Harms, M.H. Vitaterna, et al., Ehd4 is required to attain normal prepubertal testis size but dispensable for fertility in male mice, Genesis. 48 (2010) 328-342. doi:10.1002/dvg.20620.

[36] P. Arya, M.A. Rainey, S. Bhattacharyya, B.C. Mohapatra, M. George, M.R. Kuracha, et al., The endocytic recycling regulatory protein EHD1 Is required for ocular lens development, Dev. Biol. 408 (2015) 1-15. doi:10.1016/j.ydbio.2015.10.005.

[37] S. Bhattacharyya, M.A. Rainey, P. Arya, S. Dutta, M. George, M.D. Storck, et al., Endocytic recycling protein EHD1 regulates primary cilia morphogenesis and $\mathrm{SHH}$ signaling during neural tube development, Sci. Rep. 6 (2016) 20727. doi:10.1038/srep20727.

[38] M.A. Rainey, M. George, G. Ying, R. Akakura, D.J. Burgess, E. Siefker, et al., The endocytic recycling regulator EHD1 is essential for spermatogenesis and male fertility in mice., BMC Dev. Biol. 10 (2010) 37. doi:10.1186/1471-213X-10-37.

[39] J. Weischenfeldt, B. Porse, Bone marrow-derived macrophages (BMM): Isolation and applications, Cold Spring Harb. Protoc. 3 (2008) pdb.prot5080-pdb.prot5080. doi:10.1101/pdb.prot5080.

[40] T.D. Schmittgen, K.J. Livak, Analyzing real-time PCR data by the comparative CT method, Nat. Protoc. 3 (2008) 1101-1108. doi:10.1038/nprot.2008.73. 
[41] T.A. Bailey, H. Luan, E. Tom, T.A. Bielecki, B. Mohapatra, G. Ahmad, et al., A kinase inhibitor screen reveals protein kinase C-dependent endocytic recycling of ErbB2 in breast cancer cells, J. Biol. Chem. 289 (2014) 30443-30458. doi:10.1074/jbc.M114.608992.

[42] P.D. Simoncic, A. Bourdeau, A. Lee-Loy, L.R. Rohrschneider, M.L. Tremblay, E.R. Stanley, et al., T-cell protein tyrosine phosphatase (Tcptp) is a negative regulator of colony-stimulating factor 1 signaling and macrophage differentiation., Mol. Cell. Biol. 26 (2006) 4149-60. doi:10.1128/MCB.01932-05.

[43] E.R. Stanley, Factors regulating macrophage production and growth: identity of colonystimulating factor and macrophage growth factor, J. Exp. Med. 143 (1976) 631-647. doi:10.1084/jem.143.3.631.

[44] R. Wimmer, M. Baccarini, Partner exchange: Protein-protein interactions in the Raf pathway, Trends Biochem. Sci. 35 (2010) 660-668. doi:10.1016/j.tibs.2010.06.001.

[45] R.S. Liao, S. Ma, L. Miao, R. Li, Y. Yin, G. V. Raj, Androgen receptor-mediated nongenomic regulation of prostate cancer cell proliferation, Transl. Androl. Urol. 2 (2013) 187-196. doi:10.3978/j.issn.2223-4683.2013.09.07.

[46] Y.G. Yeung, E.R. Stanley, Proteomic Approaches to the Analysis of Early Events in Colony-stimulating Factor-1 Signal Transduction, Mol.Cell Proteomics. 2 (2003) 11431155. doi:10.1074/mcp.R300009-MCP200.

[47] F.J. Pixley, Macrophage migration and its regulation by CSF-1, Int. J. Cell Biol. 2012 (2012) 501962. doi:10.1155/2012/501962.

[48] D.A. Hume, K.P.A. MacDonald, Therapeutic applications of macrophage colonystimulating factor-1 (CSF-1) and antagonists of CSF-1 receptor (CSF-1R) signaling, Blood. 119 (2012) 1810-1820. doi:10.1182/blood-2011-09-379214.

[49] J.J. Jeffery, K. Lux, J.S. Vogel, W.D. Herrera, S. Greco, H.-H. Woo, et al., Autocrine inhibition of the c-fms proto-oncogene reduces breast cancer bone metastasis assessed with in vivo dual-modality imaging, Exp. Biol. Med. 239 (2014) 404-413. doi:10.1177/1535370214522588.

[50] R. Hassan, S. Suzu, M. Hiyoshi, N. Takahashi-Makise, T. Ueno, T. Agatsuma, et al., Dysregulated activation of a Src tyroine kinase Hck at the Golgi disturbs N-glycosylation of a cytokine receptor Fms, J. Cell. Physiol. 221 (2009) 458-468. doi:10.1002/jcp.21878.

[51] M. Hiyoshi, S. Suzu, Y. Yoshidomi, R. Hassan, H. Harada, N. Sakashita, et al., Interaction between Hck and HIV-1 Nef negatively regulates cell surface expression of M-CSF receptor, Blood. 111 (2008) 243-250. doi:10.1182/blood-2007-04-086017.

[52] T.S. Gomez, J. a. Gorman, a. Artal-Martinez de Narvajas, a. O. Koenig, D.D. Billadeau, Trafficking defects in WASH-knockout fibroblasts originate from collapsed endosomal and lysosomal networks, Mol. Biol. Cell. 23 (2012) 3215-3228. doi:10.1091/mbc.E12-020101 .

[53] C. Tu, C.F. Ortega-Cava, G. Chen, N.D. Fernandes, D. Cavallo-Medved, B.F. Sloane, et al., Lysosomal cathepsin B participates in the podosome-mediated extracellular matrix degradation and invasion via secreted lysosomes in v-Src fibroblasts, Cancer Res. 68 (2008) 9147-9156. doi:10.1158/0008-5472.CAN-07-5127. 
[54] D.C. Strachan, B. Ruffell, Y. Oei, M.J. Bissell, L.M. Coussens, N. Pryer, et al., CSF1R inhibition delays cervical and mammary tumor growth in murine models by attenuating the turnover of tumor-associated macrophages and enhancing infiltration by CD8(+) T cells., Oncoimmunology. 2 (2013) e26968. doi:10.4161/onci.26968.

[55] L. Hernandez, T. Smirnova, D. Kedrin, J. Wyckoff, L. Zhu, E.R. Stanley, et al., The EGF/CSF-1 paracrine invasion loop can be triggered by heregulin $\beta 1$ and CXCL12, Cancer Res. 69 (2009) 3221-3227. doi:10.1158/0008-5472.CAN-08-2871. 


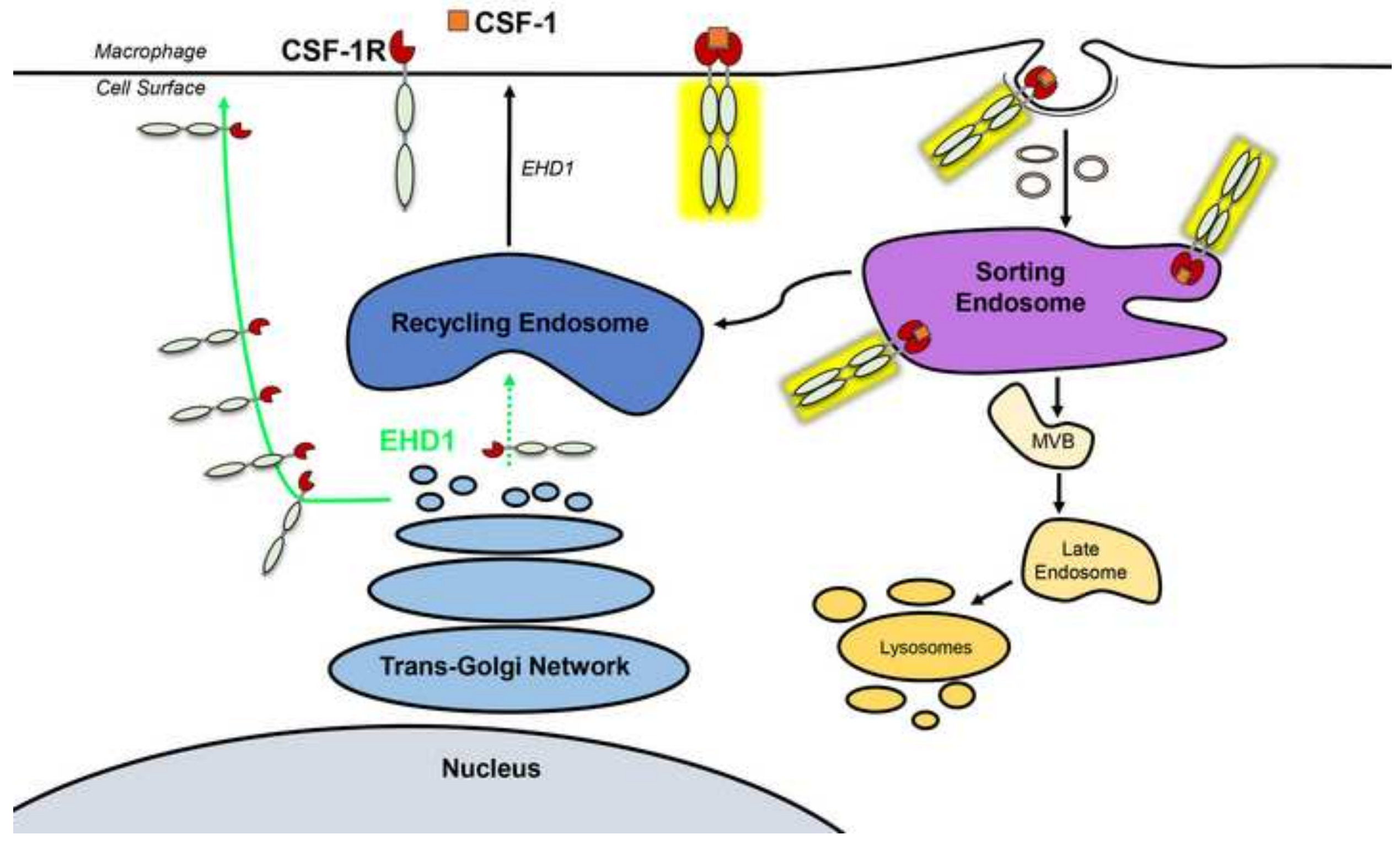


Fig. 1

A

Day 2

Day 4

Day 6
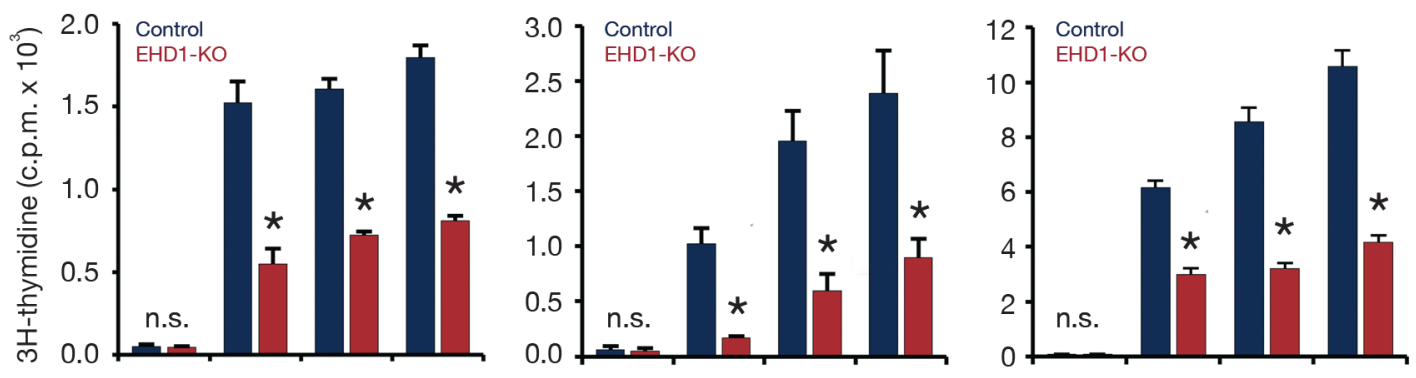

B

C

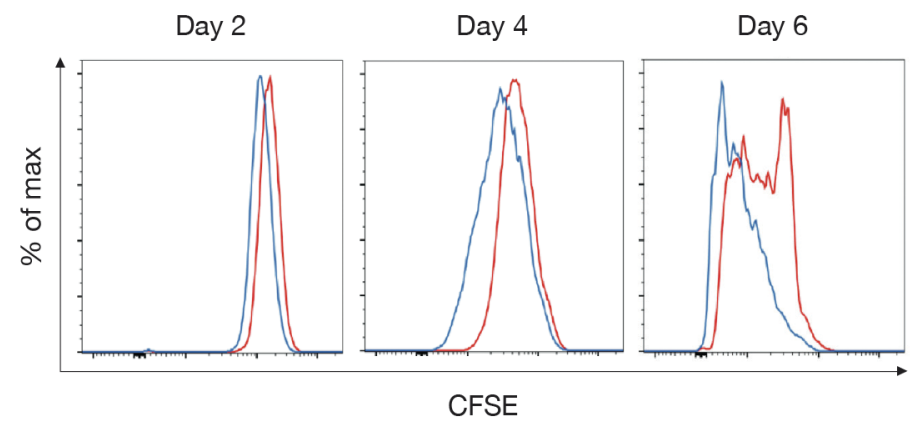

E

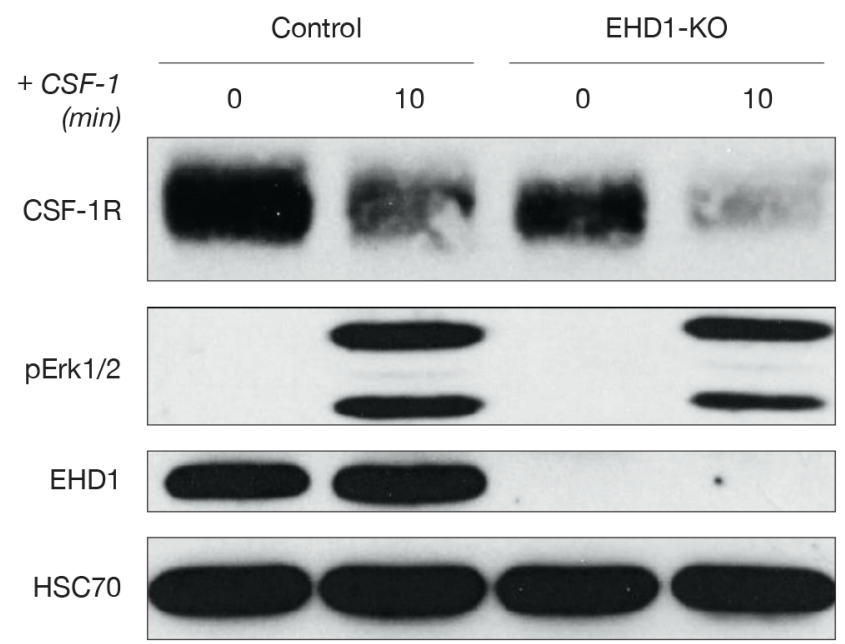

Ehd1fl/fl., Cre ${ }^{\text {ERT2 }}$

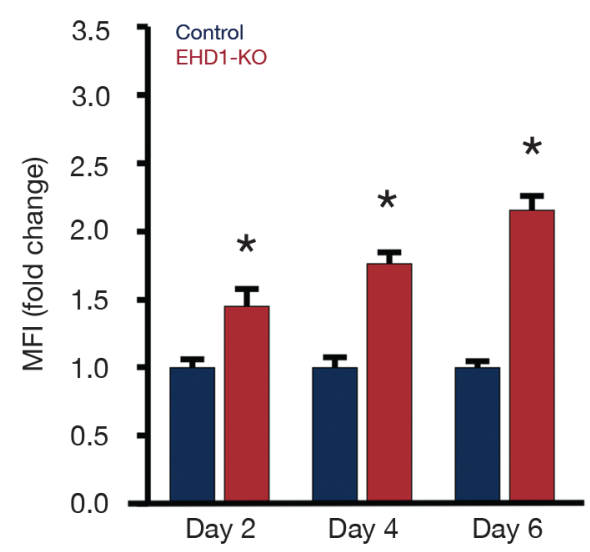

F

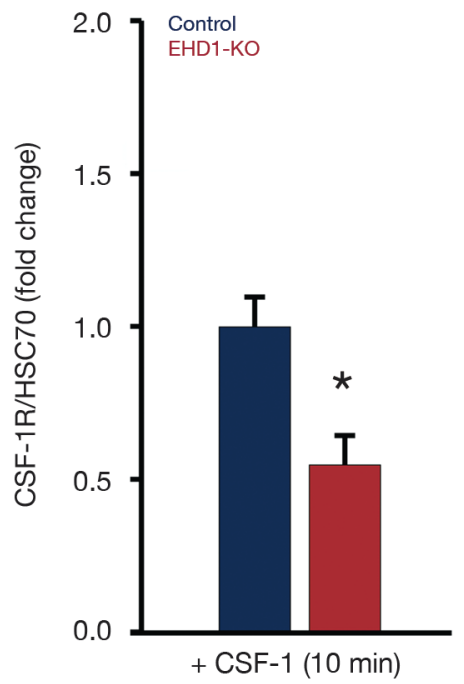


Fig. 2

A

B

CSF-1 deprivation (minutes)
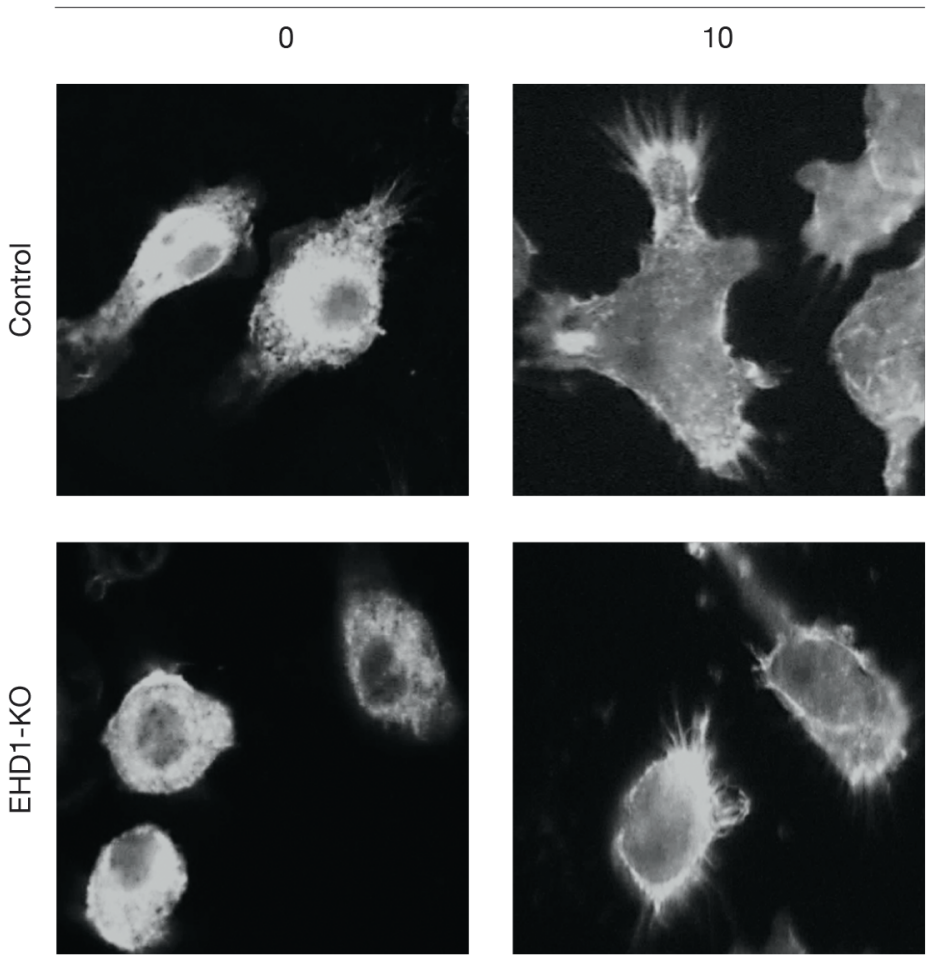

C

Control

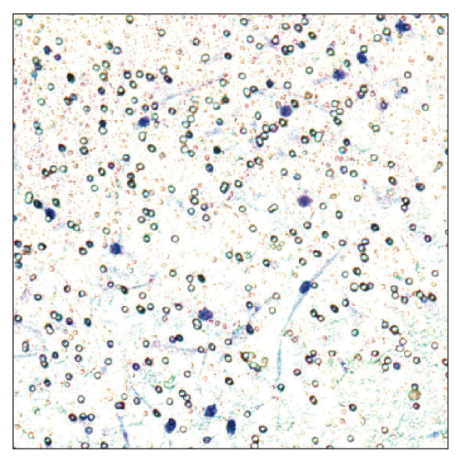

EHD1-KO

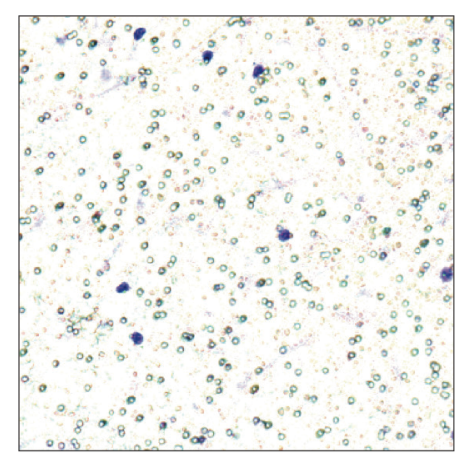

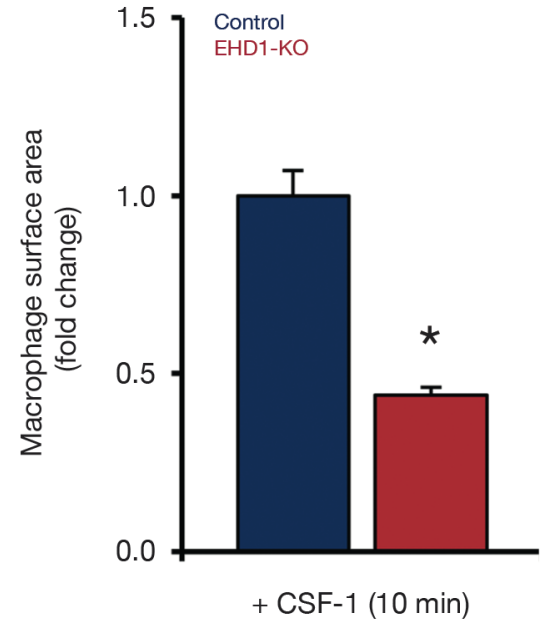

D

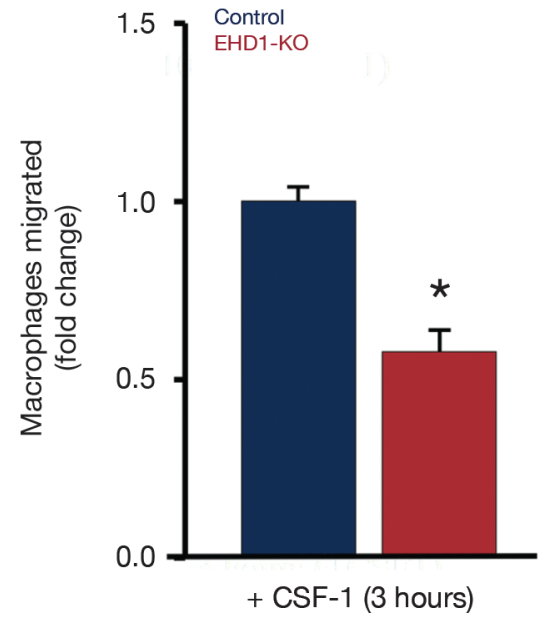


Fig. 3

A
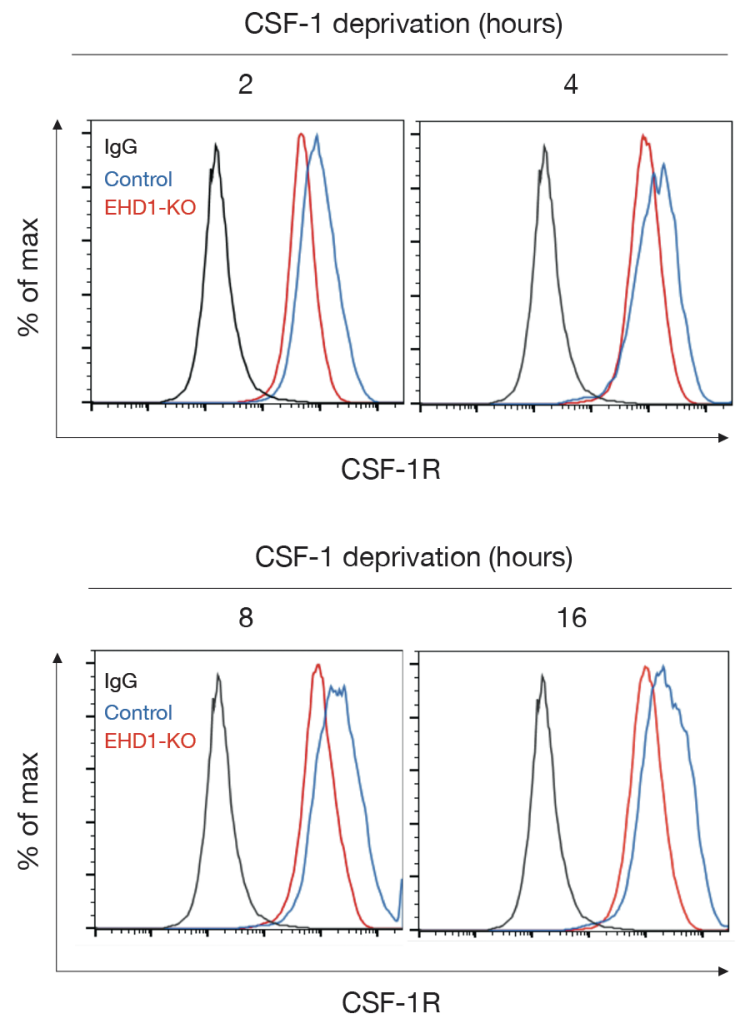

C

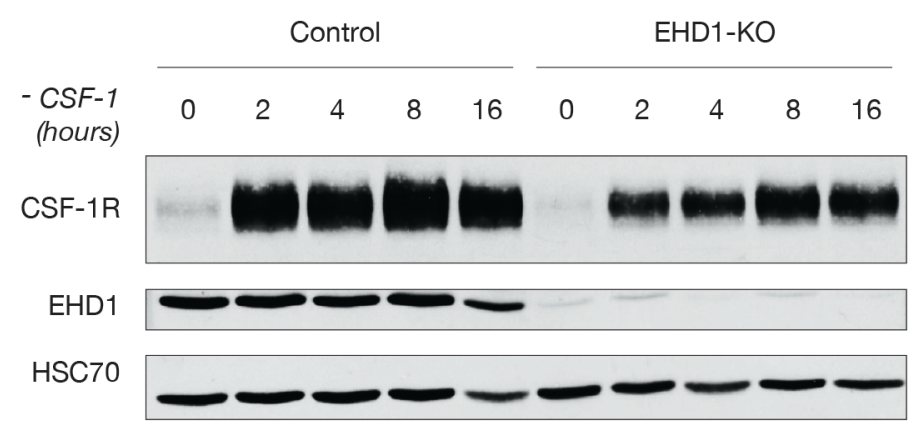

B

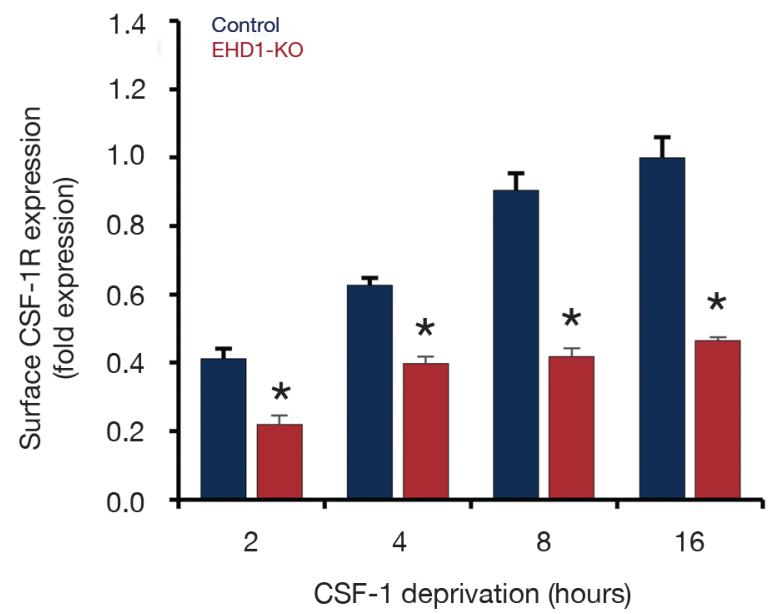

D

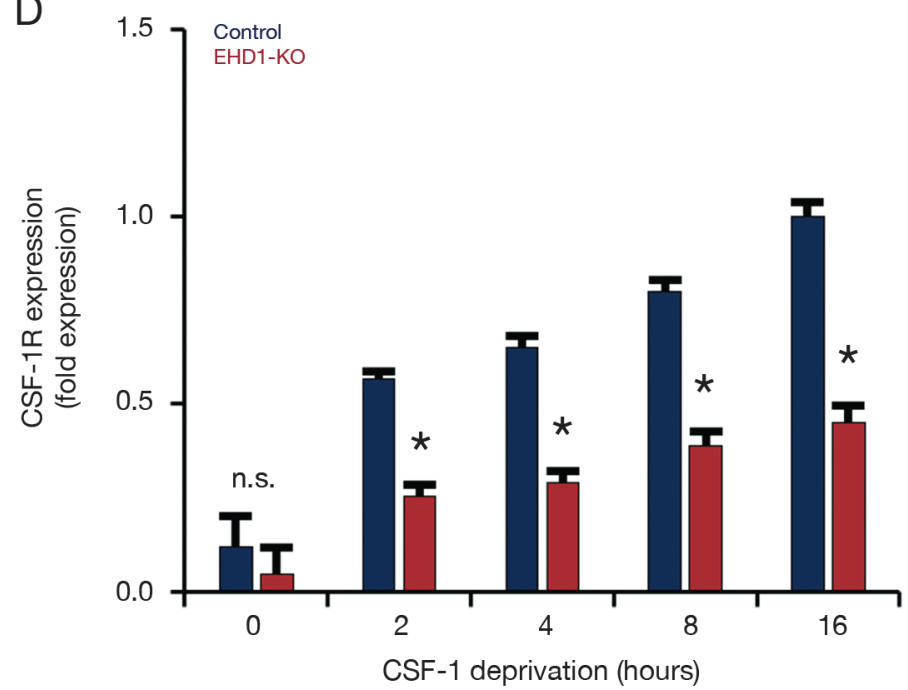


Fig. 4

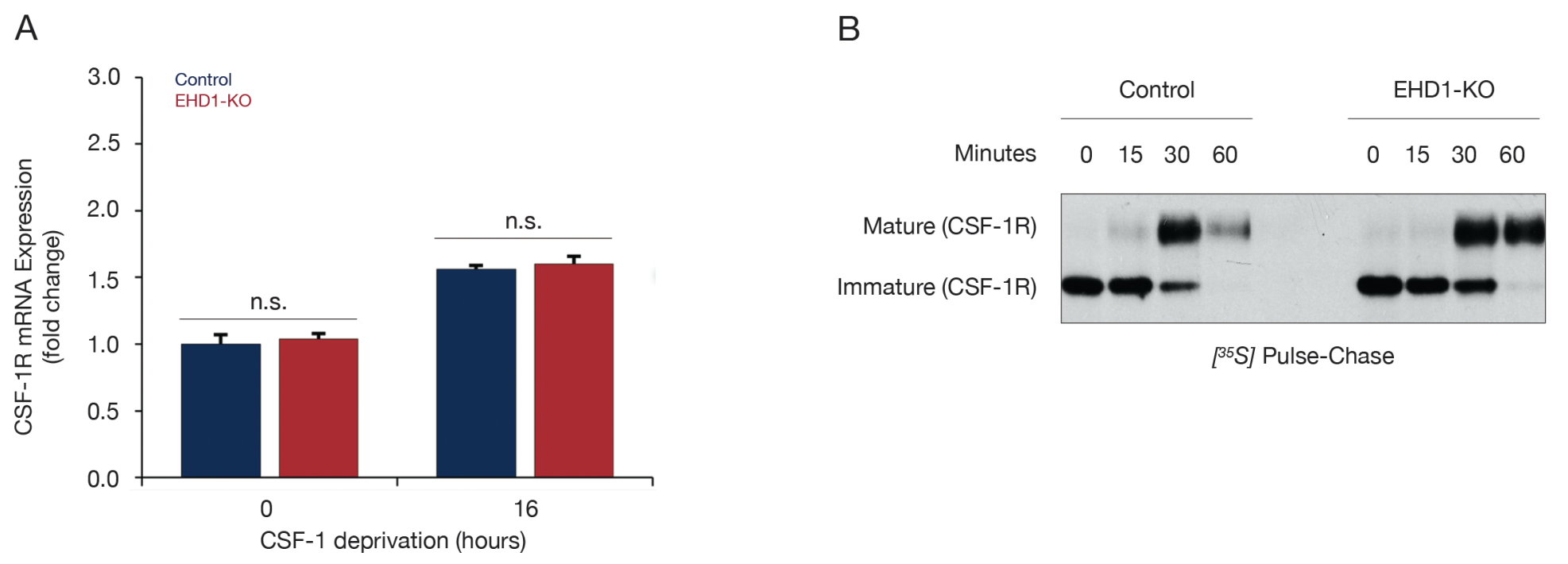


Fig. 5

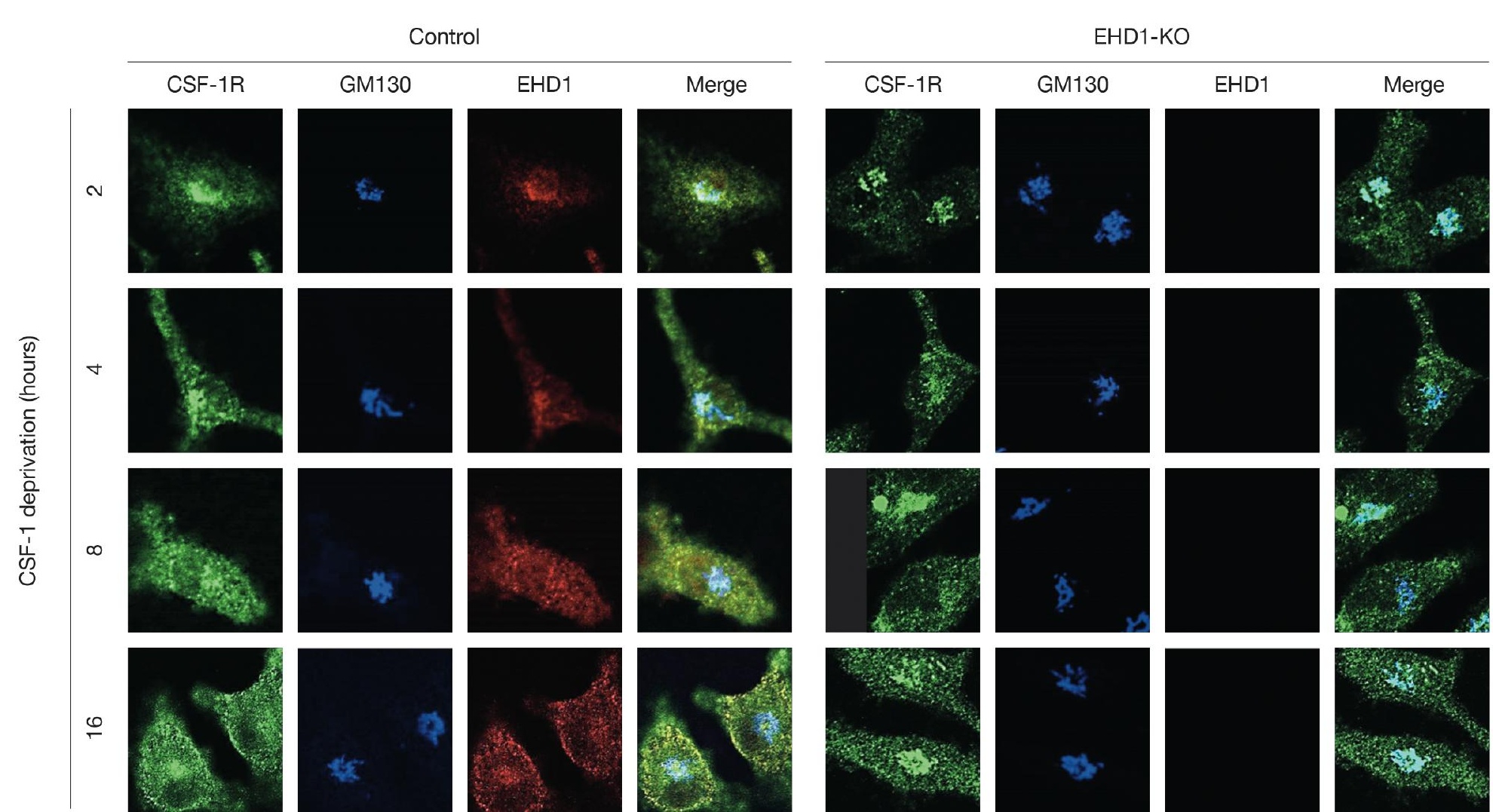


Fig. 6

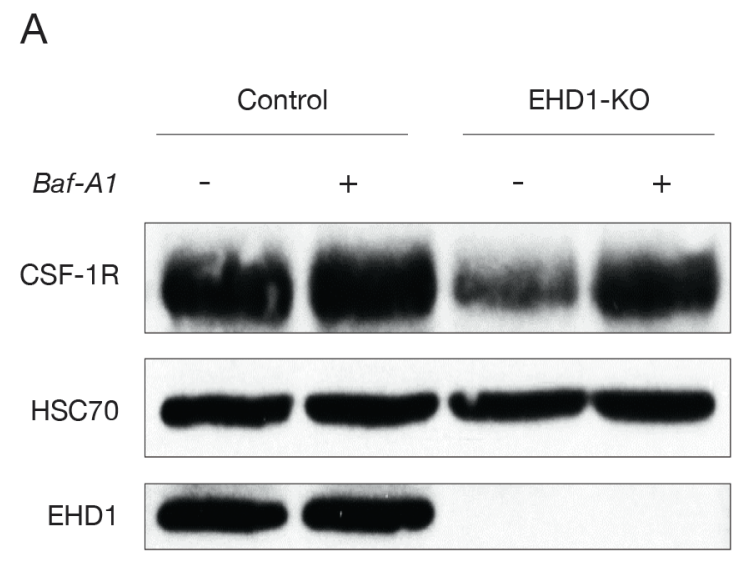

B

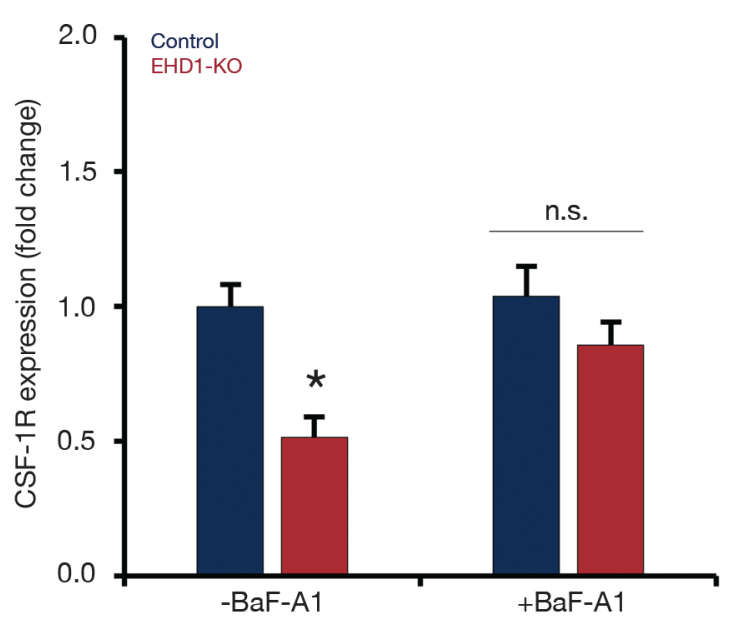

C
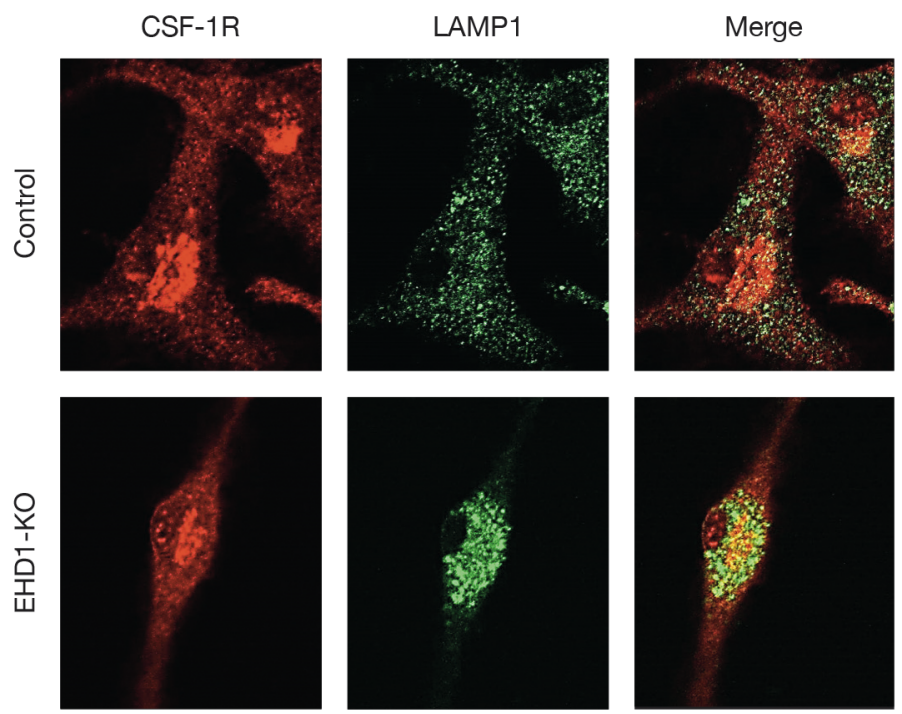

D

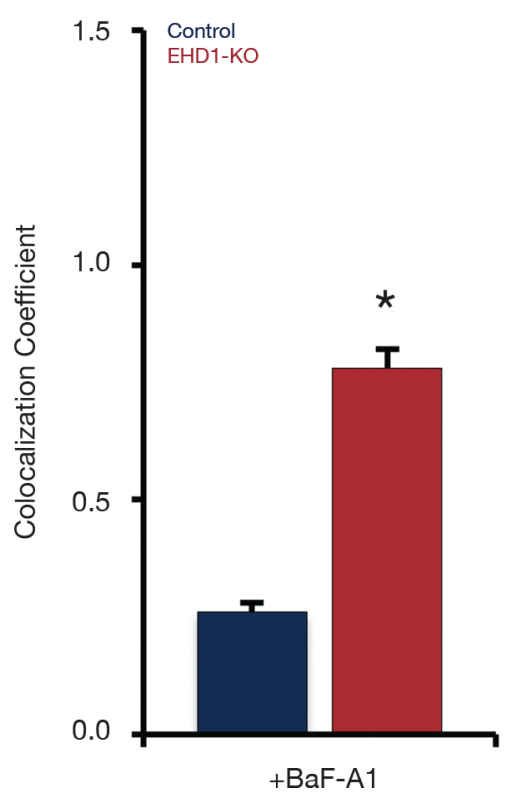

